\title{
SOME RESULTS CONCERNING THE BOUNDARY ZERO SETS OF GENERAL ANALYTIC FUNCTIONS
}

\author{
BY \\ ROBERT D. BERMAN ${ }^{1}$
}

\begin{abstract}
Two results concerning the boundary zero sets of analytic functions on the unit disk $\Delta$ are proved. First we consider nonconstant analytic functions $f$ on $\Delta$ for which the radial limit function $f^{*}$ is defined at each point of the unit circumference $C$. We show that a subset $E$ of $C$ is the zero set of $f^{*}$ for some such function $f$ if and only if it is a $\mathscr{G}_{\delta}$ that is not metrically dense in any open arc of $C$. We then give a precise version of an asymptotic radial uniqueness theorem and its converse. The constructions given in the proofs of each of these theorems employ an approximation theorem of Arakeljan.
\end{abstract}

1. Introduction. Let $\Delta=\{|z|<1\}$ and $C=\{|z|=1\}$. We assume throughout that $E$ is a subset of $C$. For $f$ a continuous function mapping $\Delta$ into the extended plane $\hat{\mathbf{C}}=\mathbf{C} \cup\{\infty\}$, let $f^{*}$ dencte its radial limit function. Thus $f^{*}(\eta)=$ $\lim _{r \rightarrow 1} f(r \eta)$ for each $\eta$ in $C$ where the limit exists (finite or infinite).

In this paper we shall apply the following theorem of N. U. Arakeljan [1] to obtain two results concerning the boundary zero sets of general analytic (and meromorphic) functions.

TheOREM A. Let $D$ be a domain in the complex plane $\mathbf{C}$ and let $K$ be a relatively closed proper subset of $D$. Then every funcrion $g$ continuous on $K$ and analytic in its interior can be uniformly approximated by an analytic function $f$ on $D$ if and only if $D^{\infty} \backslash K$ is connected and locally connected, where $D^{\infty}=D \cup\left\{z_{\infty}\right\}$ denotes the Alexandrov (one-point) compactification of $D$ and $z_{\infty}$ is the added point.

We first consider functions $f$ for which $f^{*}$ is defined at each point $\eta$ of $C$. When $f$ is a function of this kind, the set $\left\{\eta \in C: f^{*}(\eta)=0\right\}$ is a $\mathscr{G}_{\delta}$, that is, a countable intersection of open sets. Concerning bounded analytic functions, J. S. Hwang and P. Lappan [15] proved the following theorem, improving a result given by Cahill [12, Theorem 5]. Both results are based on the work of Z. Zahorski [23].

THEOREM B. There exists a nonconstant (nonvanishing) bounded analytic function $f$ on $\Delta$ having radial limits at each point of $C$ such that $E=\left\{\eta \in C: f^{*}(\eta)=0\right\}$ if and only if $E$ is a $\mathscr{G}_{\delta}$ of measure 0 .

Received by the editors October 8, 1984 and, in revised form, April 15, 1985.

1980 Mathematics Subject Classification. Primary 30D40.

${ }^{1}$ The author gratefully acknowledges support from a Wayne State University Faculty Research Grant. 
In [8], the author gave the corresponding characterization for inner functions, that is, bounded analytic functions $f$ for which $\left|f^{*}\right|=1$ a.e. It was shown that $E=\{\eta$ $\left.\in C: f^{*}(\eta)=0\right\}$ for an inner function $f$ having radial limits at each point of $C$ if and only if $E$ is a $\mathscr{G}_{\delta}$ contained in an $\mathscr{T}_{\sigma}$ (a countable union of closed sets) of measure 0. The main result of $\S 2$ is the analogous result for general analytic functions.

THEOREM 1.1. There exists a nonconstant analytic (or meromorphic) function fon $\Delta$ having radial limits at each point of $C$ for which $E=\left\{\eta \in C: f^{*}(\eta)=0\right\}$ if and only if $E$ is a $\mathscr{G}_{\delta}$ that is not metrically dense in any open arc of $C$.

Recall that $E$ is said to metrically dense in a (nonempty) open $\operatorname{arc} A$ if $E \cap B$ has positive outer measure for every open subarc $B$ of $A$. Theorem 1.1 sharpens a result given in [10]. Along with Theorem $\mathrm{A}$, its proof relies on results and methods used in [10] and in recent work of C. L. Belna, P. Colwell and G. Piranian [5] in which the boundary zero sets of the class of all inner functions are characterized.

Our second application concerns radial approach to zero at a specified asymptotic rate. K. F. Barth and W. J. Schneider [4], and K. F. Tse [22] proved an asymptotic radial uniqueness theorem for arbitrary analytic functions on $\Delta$. Along with the converse given by $\mathrm{P}$. Gauthier (see [22, Theorem B]), this result may be stated as follows.

THEOREM C. Let $\mu$ be a positive decreasing function on $[0,1)$ such that $\lim _{r \rightarrow 1} \mu(r)$ $=0$. Then there exists a nonconstant analytic (or meromorphic) function $f$ on $\Delta$ such that $f(r \eta)=O[\mu(r)]$ for each $\eta \in E$ if and only if $E$ is of first category.

In $\S 3$ we prove a more precise version of Theorem $C$.

THEOREM 1.2. Let $\mu$ be a positive decreasing function on $[0,1)$ such that $\lim _{r \rightarrow 1} \mu(r)=0$. Then there exists a nonconstant analytic (or meromorphic) function $f$ on $\Delta$ such that $E=\{\eta \in C: f(r \eta)=O[\mu(r)]\}$ if and only if $E$ is first-category $\mathscr{T}_{\sigma}$ set.

Necessity in Theorem 1.2 follows from an elementary proof of the BarthSchneider-Tse theorem given in [6]. The proof of sufficiency again uses Theorem A and the methods of $\S 2$.

In $\S 4$, analogues for continuous subharmonic functions are given. These are easily derived from previous results for subharmonic functions (see, for example, [9]), and the theorems for analytic functions stated in this section. In the final section, $\$ 5$, we state some open problems.

2. Proof of Theorem 1.1. We start by establishing notation and proving some elementary results. For $f$ a meromorphic function on $\Delta$, let $C_{\rho}(f, \eta)$ denote the radial cluster set of $f$ at $\eta \in C$. Thus, by definition,

$$
C_{\rho}(f, \eta)=\bigcap_{1}^{\infty} \overline{\{f(r \eta):(n-1) / n \leqslant r<1\}}, \quad \eta \in C .
$$

Here and in the sequel, $\bar{W}$ (respectively int $W$ ) denotes the closure (respectively interior) of a subset $W$ of $\mathbf{C}$. Let $Z_{\rho}(f)=\left\{\eta \in C: 0 \in C_{\rho}(f, \eta)\right\}$. 
Proposition 2.1. The set $Z_{\rho}(f)$ is a $\mathscr{G}_{\delta}$.

Proof. For each positive integer $n$, let

$$
F_{n}=\{\eta \in C:|f(r \eta)| \geqslant 1 / n \text { for }(n-1) / n \leqslant r<1\} .
$$

By the continuity of $f$, each $F_{n}$ is closed. Furthermore,

$$
C \backslash Z_{\rho}(f)=\bigcup_{n=1}^{\infty} F_{n} .
$$

Thus $Z_{\rho}(f)$ is the complement of an $\mathscr{T}_{\sigma}$, that is, a $\mathscr{G}_{\delta}$. This completes the proof.

If $\eta \in C$ and $C_{\rho}(f, \eta) \neq \hat{\mathbf{C}}$, then $C_{\rho}(f, \eta)$ is said to be subtotal.

DEFINITION 2.1. Let $\mathscr{M}_{S}$ be the class of all meromorphic functions $f$ on $\Delta$ for which $C_{\rho}(f, \eta)$ is subtotal for a residual set of $\eta$ in $C$ (that is, the complement of a first-category set).

Observe that if $\eta \in C$ and $f^{*}(\eta)$ is defined, then $C_{\rho}(f, \eta)=\left\{f^{*}(\eta)\right\}$ so that $C_{\rho}(f, \eta)$ is subtotal. This leads to the next result.

PROPOSITION 2.2. If $f$ is a meromorphic function on $\Delta$ such that $f^{*}(\eta)$ is defined for each $\eta$ in $C$, then $f \in \mathscr{M}_{S}$ and $Z_{\rho}(f)=\left\{\eta \in C: f^{*}(\eta)=0\right\}$ is a $\mathscr{G}_{\delta}$.

The next theorem is a generalization of the Lusin-Privalov radial uniqueness theorem (see [14, Theorem 8.3(i)]) proved by E. F. Collingwood and, independently, by F. Bagemihl and W. Seidel.

THEOREM D. Let $f$ be a meromorphic function on $\Delta$ and let $A$ be an open arc of $C$. If $C_{\rho}(f, \eta)$ is subtotal for a set of $\eta$ of second category in $A$, and $b \in C_{\rho}(f, \eta)$ for a set of $\eta$ which is metrically dense in $A$, then $f \equiv b$.

The following is a corollary of Theorem D and Proposition 2.2 that completes the proof of necessity in Theorem 1.1.

COROLlaRY 2.1. If $f$ is nonconstant meromorphic function on $\Delta$ such that $f^{*}(\eta)$ is defined for each $\eta$ in $C$, then $Z_{\rho}(f)=\left\{\eta \in C: f^{*}(\eta)=0\right\}$ is a $\mathscr{G}_{\delta}$ that is not metrically dense in any arc of $C$.

In [10], it is shown that if $E$ is a subset of $C$ that is not metrically dense in any open arc of $C$, then there exists a nonconstant analytic function $f$ on $\Delta$ whose modulus has radial limits at each point of $C$ such that $f^{*}(\eta)=0$ for $\eta \in E$. We shall now refine this result to prove sufficiency in Theorem 1.1. Our procedure will be similar in its use of the following lemma proved in [7].

LEMMA 2.1. If for every open arc $A$ of $C$, the set $E$ is not both metrically dense and of second category in $A$, then there exists a closed subset $F$ of $C$ such that $E \backslash F$ has zero measure and $E \cap F$ is of first category.

Here, the set $E$ is a $\mathscr{G}_{\delta}$ that is not metrically dense in any open $\operatorname{arc}$ of $C$, and the lemma insures the existence of a closed set $F$ such that $E \backslash F$ is a $G_{\delta}$ of measure 0 , and $E \cap F$ is a first-category $\mathscr{G}_{\delta}$ (in fact, a nowhere-dense $\mathscr{G}_{\delta}$ ). The set $E \backslash F$ is handled using Theorem B. For $E \cap F$, we shall use the following proposition. 
Proposition 2.3. If $E$ is a first-category $\mathscr{G}_{\delta}$, then there exists an analytic function $f$ on $\Delta$ such that $f^{*}$ is defined and finite at each point of $C$ and $E=\{\eta \in C$ : $\left.f^{*}(\eta)=0\right\}$.

In order to give the proof, we shall need some additional notation and a lemma. For each $\eta \in C$ and $r \in(0,1)$, let

$$
R(\eta)=\{z \in \mathbf{C} \backslash\{0\}: z /|z|=\eta\} \cup\{0\},
$$

and let $d(\eta, r)$ be the open disk in $\Delta$ of radius $r$ whose circumference is tangent to $C$ at $\eta$. In addition, for any subset $E$ of $C$, let $R(E)=\bigcup_{\eta \in E} R(\eta)$ and $d(E, r)=$ $\bigcup_{\eta \in E} d(\eta, r)$. If $A$ is any proper open arc of $C$, let $S_{A}$ be the open sector of $\Delta$ defined by

$$
S_{A}=\{z \in \Delta \backslash\{0\}: z /|z| \in A\} .
$$

Let $\arg \eta, \eta \in A$, be a continuous argument of the identity function on $A$ and let $\mathbf{Z}$ denote the integers.

Supose now that $V$ is a closed nowhere-dense subset of $C$ and $A$ a proper open arc of $C$. Select $\left(\eta_{j}\right)_{j \in \mathbf{Z}} \subset A \backslash V$ such that $\arg \eta_{j}$ is an increasing function of $j$ in $\mathbf{Z}$ and $\lim _{j \rightarrow \pm \infty} \eta_{j}$ are the endpoints of $A$. For each $j \in \mathbf{Z}$, let $F_{j}$ be the closed nowhere-dense subset of $C$ consisting of the intersection of $V$ with the open $\operatorname{arc} A_{j}$ having endpoints $\eta_{j}$ and $\eta_{j+1}$. Given $\varepsilon \in(0,1)$, choose $\left(r_{j}\right)_{j \in \mathbf{Z}}$ so that $\lim _{j \rightarrow \pm \infty} r_{j}$ $=0$, and for each $j, r_{j} \in(0, \varepsilon)$ and $d\left(F_{j}, r_{j}\right) \subset S_{A_{j}}$.

LemMA 2.2. Let $\varepsilon \in(0,1)$. There exists an analytic function $f$ on $\Delta$ such that

(i) $f^{*}$ exists at each point of $C$,

(ii) $V \cap A=\left\{\eta \in C: f^{*}(\eta)=0\right\}$,

(iii) $|f(z)-1|<\varepsilon, z \in \Delta \backslash \bigcup_{j \in \mathbf{Z}} d\left(F_{j}, r_{j}\right)$, and

(iv) $|f(z)| \leqslant 1+\varepsilon, z \in \Delta \cap R(A \cap V)$.

Proof. For each $j \in \mathbf{Z}$, let

$$
D_{j}=\left[\mathbf{C} \backslash R\left(F_{j}\right)\right] \cup \Delta, \quad K_{j}=\left[D_{j} \backslash d\left(F_{j}, r_{j}\right)\right] \cup\left[R\left(F_{j}\right) \cap d\left(F_{j}, r_{j}\right)\right],
$$

and define $g_{j}$ by

$$
g_{j}(z)= \begin{cases}0, & z \in D_{j} \backslash d\left(F_{j}, r_{j}\right), \\ \ln \frac{2 r_{j}}{1-|z|}, & z \in R\left(F_{j}\right) \cap d\left(F_{j}, r_{j}\right) .\end{cases}
$$

Let $t_{0}>0$ such that $\exp (t)-1 \leqslant 2 t$ for all $t \in\left[0, t_{0}\right]$ and assume without loss of generality that $\varepsilon<t_{0}$. Note that for each $j$, the set $K_{j}$ is a relatively closed subset of the domain $D_{j}$ such that $D_{j}^{\infty} \backslash K_{j}$ is connected and locally connected, and $g_{j}$ is continuous on $K_{j}$ and analytic in its interior. Therefore, by Theorem A there exists for every $j \in \mathbf{Z}$ an analytic function $f_{j}$ on $D_{j}$ such that

$$
\left|f_{j}(z)-g_{j}(z)\right|<\varepsilon / 2^{(|j|+2)}, \quad z \in K_{j} .
$$


From the definition of $g_{j}$ and the domain $D_{j}$, it follows that

$$
\left|f_{j}(z)\right|<\varepsilon / 2^{(|j|+2)}, \quad z \in D_{j} \backslash d\left(F_{j}, r_{j}\right),
$$

and $\left.f_{j}\right|_{\Delta}$ has radial limits at each point of $C$ for every $j \in \mathbf{Z}$.

Define $h(z)=\sum_{j \in \mathbf{Z}} f_{j}(z)$ for $z \in \Delta$. By (2.2) and the choice of $\left(r_{j}\right)_{j \in \mathbf{Z}}$ so that $d\left(F_{j}, r_{j}\right) \subset S_{A_{j}}$ for each $j$ and $\lim _{j \rightarrow \pm \infty} r_{j}=0$, we see that the sum is uniformly convergent on compact subsets and each radius of $\Delta$,

$$
\left|h(z)-f_{k}(z)\right| \leqslant \sum_{j \neq k}\left|f_{j}(z)\right| \leqslant \varepsilon\left(\frac{1}{2}-\frac{1}{2^{|k|+2}}\right), \quad z \in \Delta \cap R\left(A_{k}\right), k \in \mathbf{Z},
$$

and $h^{*}$ is defined at each point of $C$ with

$$
\left|h^{*}(\eta)\right| \leqslant \varepsilon / 2, \quad \eta \in C \backslash(A \cap V),
$$

and

$$
\lim _{r \rightarrow 1} \operatorname{Re} h(r \eta)=+\infty, \quad \eta \in A \cap V .
$$

It also follows from (2.1) and (2.4) that

$$
\operatorname{Re} h(z) \geqslant-\varepsilon / 2, \quad z \in \Delta \cap R(A \cap V) .
$$

Let $f=\exp (-h)$. Since $h^{*}$ is defined at each point of $C$ and (2.5) and (2.6) hold, it follows that $f^{*}$ is defined at each point of $C$ with $V \cap A=\left\{\eta \in C: f^{*}(\eta)=0\right\}$. Furthermore, (iii) and (iv) follow from (2.3) and (2.7) together with the inequalities

$$
|\exp (z)-1| \leqslant \exp (|z|)-1 \leqslant 2|z|, \quad|z| \leqslant t_{0} .
$$

This completes the proof of the lemma.

We turn now to the proof of Proposition 2.3.

Proof. We put aside the trivial case and assume that $E \neq \varnothing$. Since $E$ is a $\mathscr{G}_{\delta}$ of first category, it is contained in a closed nowhere-dense set $V$. (Recall that a $\mathscr{G}_{\delta}$ that is dense in an arc is residual in it.) Also, there exists a descending sequence $\left(U_{n}\right)_{1}^{\infty}$ of open subsets of $C$ such that $E=\bigcap_{1}^{\infty} U_{n}$. For each $n$, we have $U_{n}=\bigcup_{k} A_{n k}$, where $\left(A_{n k}\right)$ is a finite or infinite sequence of mutually disjoint open arcs each having nonempty intersection with $E$. Furthermore, for any pair of allowed positive integers $n$ and $k$, we have $A_{n+1, k} \subseteq A_{n j}$ for some positive integer $j$.

We proceed by induction to define $\left(A_{n k j}\right)_{j \in \mathbf{Z}}\left(F_{n k j}\right)_{j \in \mathbf{Z}}$ and $\left(r_{n k j}\right)_{j \in \mathbf{Z}}$ relative to the arc $A_{n k}$ for all such arcs. Here and in the remainder of the proof, we use subscripted notation corresponding to that preceding Lemma 2.2.

$n=1$. Choose $\left(A_{1 k j}\right)_{j \in \mathbf{Z}},\left(F_{1 k j}\right)_{j \in \mathbf{Z}}$ and $\left(r_{l k j}\right)_{j \in \mathbf{Z}}$ relative to $A_{l k}$ as preceding Lemma 2.2 with $\varepsilon=1 / 2^{(1+k)}$ for each $k$.

Inductive hypothesis. Assume that $n \geqslant 1$ and $\left(A_{n k j}\right)_{j \in \mathbf{Z}},\left(F_{n k j}\right)_{j \in \mathbf{Z}}$ and $\left(r_{n k j}\right)_{j \in \mathbf{Z}}$ have been selected relative to $A_{n k}$ as preceding Lemma 2.2 with $\varepsilon=1 / 2^{(n+k)}$ for each $k$. Assume also that if $1 \leqslant n^{\prime} \leqslant n, A_{n k} \subseteq A_{n^{\prime} k^{\prime}}$ and $\eta \in A_{n k} \cap V$, then the sets $F_{n k j}$ and $F_{n^{\prime} k^{\prime} j^{\prime}}$ that contain $\eta$ along with the associated $r_{n k j}$ and $r_{n^{\prime} k^{\prime} j^{\prime}}$ satisfy $F_{n k j} \subseteq F_{n^{\prime} k^{\prime} j^{\prime}}$ and $r_{n k j} \leqslant r_{n^{\prime} k^{\prime} j^{\prime}}$. 
$n+1$. For each arc $A_{n+1, k}$, there exists a unique positive integer $k^{\prime}$ such that $A_{n+1, k} \subseteq A_{n k^{\prime}}$. Noting the simple structure of the sets $F_{n k^{\prime} j^{\prime}} \cap A_{n+1, k}$ for $j^{\prime} \in \mathbf{Z}$, the sets $\left(A_{n+1, k j}\right)_{j \in \mathbf{Z}}$ and $\left(F_{n+1, k j}\right)_{j \in \mathbf{Z}}$ can be constructed as preceding Lemma 2.2 so that each $F_{n+1, k j}$ is contained in some $F_{n k^{\prime} j^{\prime}}$. Then choose $\left(r_{n+1, k j}\right)_{j \in \mathbf{Z}}$ in $\left(0,1 / 2^{(n+k+1)}\right)$ with $r_{n+1, k j} \leqslant r_{n k^{\prime} j^{\prime}}$ and $\lim _{j \rightarrow \pm \infty} r_{n+1, k j}=0$.

It is verified without difficulty that the inductive hypothesis holds with $n+1$ replacing $n$, and the induction is completed. Therefore, there exist $\left(A_{n k j}\right)_{j \in \mathbf{Z}}$, $\left(F_{n k j}\right)_{j \in \mathbf{Z}}$ and $\left(r_{n k j}\right)_{j \in \mathbf{Z}}$ such that the inductive hypothesis holds for all allowed positive integers $n$ and $k$. The following observations will be used.

(I) $\left[\Delta \backslash \cup_{k} \cup_{j \in \mathbf{Z}} d\left(F_{n k j}, r_{n k j}\right)\right]_{n=1}^{\infty}$ forms a monotone nondescending sequence of sets such that for every compact subset $K$ of $\Delta$, only finitely many have nonempty intersection with $K$.

(II) If $n$ is a positive integer and $\eta \notin U_{n}$, then $\Delta \cap R(\eta)$ does not intersect any of the sets $\bigcup_{k} \cup_{j \in \mathbf{Z}} d\left(F_{m k j}, r_{m k j}\right)$ for $m \geqslant n$ since $\left(U_{l}\right)_{1}^{\infty}$ is a descending sequence and each set $\bigcup_{j \in \mathbf{Z}} d\left(F_{m k j}, r_{m k j}\right)$ is contained in the sector $S_{A_{m k}}$.

(III) If $n$ is a positive integer and $\eta \in U_{n}$, then for $1 \leqslant m \leqslant n$, either $\eta \in V \cap U_{m}$ in which case $\eta \in F_{m k j}$ for some $k$ and $j$, or else $\eta \notin V \cap U_{m}$ and $r \eta \in$ $\Delta \backslash \cup_{k} \cup_{j \in \mathbf{Z}} d\left(F_{m k j}, r_{m k j}\right)$ for $r \in[0,1)$ sufficiently close to 1 .

For each pair of positive integers $n$ and $k$, let $f_{n k}$ be as in Lemma 2.2 relative to $\varepsilon=1 / 2^{(n+k)}$ with $F_{j}$ and $r_{j}$ replaced by $F_{n k j}$ and $r_{n k j}$ respectively. Let $\left(h_{m}\right)_{1}^{\infty}$ be an enumeration of the functions $f_{n k}+1 / 2^{(n+k)}$. Define $f=\prod_{1}^{\infty} h_{m}$. From (I), we see that $f$ is uniformly product convergent on compact subsets of $\Delta$ and therefore analytic. If $\eta \in C \backslash E$, then $\eta$ is contained in at most finitely many $U_{n}$, and it follows from (II), (III) and (iii) of Lemma 2.2 that $f$ is uniformly product convergent on the radius $\Delta \cap R(\eta)$. From (ii) of the lemma and the definition of the $h_{m}$, we have $h_{m}^{*}$ is defined and nonvanishing at each point of $C$, so we conclude that $f^{*}$ is defined and not zero at each point of $C \backslash E$. Finally, since $\lim _{m \rightarrow \infty} h_{m}^{*}(\eta)=0$ for each $\eta \in E$, it follows from (iv) of the lemma that $f^{*}(\eta)=0$ for all $\eta \in E$. The proof of Proposition 2.3 is thereby completed.

Proof of Sufficiency in Theorem 1.1. Putting aside the trivial case when $E=\varnothing$, let $E$ be a nonempty $\mathscr{G}_{\delta}$ that is not metrically dense in any open arc of $C$. By Lemma 2.1 there exists a closed set $F$ such that $E \backslash F$ is a $\mathscr{G}_{\delta}$ of measure 0 and $F \cap E$ is a first-category $\mathscr{G}_{\delta}$. By Theorem $\mathrm{B}$, there is a bounded analytic function $g$ on $\Delta$ having radial limits at each point of $C$ and for which $E \backslash F=\left\{\eta \in C: g^{*}(\eta)=0\right\}$. From Proposition 2.3, there exists an analytic function $h$ on $\Delta$ such that $h^{*}$ is defined and finite at each point of $C$ with $E \cap F=\left\{\eta \in C: h^{*}(\eta)=0\right\}$. Then $f=g h$ is an analytic function on $\Delta$ having radial limits at each point of $C$ for which $E=\{\eta \in C$ : $\left.f^{*}(\eta)=0\right\}$. Since $\varnothing \neq E \neq C$, the function $f$ is nonconstant. The proof is thereby completed.

3. Proof of Theorem 1.2. For necessity, we restate the proof given in [6]. Let $f$ be a nonconstant meromorphic function on $\Delta$. Suppose that $E=\{\eta \in C: f(r \eta)=$ $O[\mu(r)]\}$. Then $E=\bigcup_{1}^{\infty} E_{n}$, where

$$
E_{n}=\{\eta \in C:|f(r \eta)| \leqslant n \mu(r) \text { for } 1-1 / n \leqslant r<1\}
$$


for each $n$. By the continuity of $f$, each $E_{n}$ is closed. If int $E_{n} \neq \varnothing$ for some $n$, say $A$ is an open arc contained in int $F_{n}$, then by the definition of $E_{n}$ the function $f$ is continuously 0 at each point of $A$. Then the Schwarz reflection principle and the identity theorem imply that $f \equiv 0$, a contradiction. We conclude that each $E_{n}$ is nowhere dense, and necessity follows.

Sufficiency. If $E=\varnothing$, then take $f(z)=\exp (z)$. Suppose now that $E$ is a nonempty first-category $\mathscr{T}_{\sigma}$. Then $E=\bigcup_{1}^{\infty} E_{n}$, where $\left(E_{n}\right)_{1}^{\infty}$ is a sequence of mutually disjoint closed nowhere-dense sets (see, for example, [8, Theorem 3.2]) and there exists a decreasing sequence $\left(r_{n}\right)_{1}^{\infty}$ in $(0,1 / 2)$ converging to 0 such that $\left[\overline{d\left(E_{n}, r_{n}\right)}\right]_{n=1}^{\infty}$ is a sequence of mutually disjoint compact sets. Define

$$
K=\left[\Delta \backslash \bigcup_{n=1}^{\infty} d\left(E_{n}, r_{n}\right)\right] \cup[R(E) \cap \Delta] .
$$

The following properties can be verified.

(i) The set $K$ is a relatively closed proper subset of $\Delta$ such that $\Delta^{\infty} \backslash K$ is connected and locally connected.

(ii) For every $\eta \in E$, there exists a positive integer $n$ such that $d\left(E_{n}, r_{n}\right) \cap R(\eta)$ $\subset K$.

(iii) If $\eta \notin E$, then there exists a sequence $\left(z_{n}\right)_{1}^{\infty}$ in $R(\eta) \cap \Delta$ converging to $\eta$ such that $z_{n} \in$ int $K$ for all positive integers $n$.

Assume without loss of generality that $\mu$ is continuous and let

$$
g(z)= \begin{cases}0, & z \in \Delta \backslash \bigcup_{n=1}^{\infty} d\left(E_{n}, r_{n}\right), \\ \ln \frac{1}{\mu(|z|)}-\ln \frac{1}{\mu\left(1-2 r_{n}\right)}, & z \in d\left(E_{n}, r_{n}\right) \cap R\left(E_{n}\right), n=1,2, \ldots\end{cases}
$$

Then $G$ is continuous on $K$ and analytic (identically 0 ) in int $K$. By property (i) and Theorem A, there exists a function $h$ analytic on $\Delta$ such that

$$
|h(z)-g(z)|<1, \quad z \in K .
$$

Using properties (ii) and (iii), there exists $\delta_{n} \in(0, \infty)$ such that for all $r \in[0,1)$ we have

$$
\operatorname{Re} h(r \eta) \geqslant-\delta_{n}+\ln \frac{1}{\mu(r)}, \quad \eta \in E_{n},
$$

for $n=1,2, \ldots$, and

$$
\liminf _{r \rightarrow 1} \operatorname{Re} h(r \eta)<+\infty, \quad \eta \in C \backslash E .
$$

Let $f=\exp (-h)$. Then $f$ is a nonvanishing analytic function on $\Delta$, and (3.2) and (3.3) insure that $f$ has the required properties. This competes the proof.

4. Subharmonic analogues. For $u(\not \equiv-\infty)$ subharmonic on $\Delta$, the radial limit function (taking values in $\hat{\mathbf{R}}=\mathbf{R} \cup\{ \pm \infty\}$ ) is denoted by $u^{*}$. Since every analytic function $f \neq 0$ corresponds to a continuous subharmonic function $u=\log |f|$, the 
natural analogue of the zeros of $f^{*}$ are the $-\infty$-points of $u^{*}$. It turns out that many boundary zero set results for analytic functions have subharmonic analogues. M. G. Arsove [2] and P. J. Rippon [20] were the first to give analogues of the Lusin-Privalov and Barth-Schneider-Tse theorems. These along with other results can be found in [9]. Putting together the results of the previous sections with ones given in [9], we arrive at the following theorems.

THEOREM 4.1. There exists a continuous subharmonic function $u \leqslant 0$ on $\Delta$ such that $u^{*}$ exists at each point of $C$ and $E=\left\{\eta \in C: u^{*}(\eta)=-\infty\right\}$, if and only if $E$ is a $\mathscr{G}_{\delta}$ of measure 0 .

THEOREM 4.2. There exists a continuous subharmonic function $u \leqslant 0$ such that $u^{*}$ is defined at each point of $C, u^{*}=0$ a.e. and $E=\left\{\eta \in C: u^{*}(\eta)=-\infty\right\}$, if and only if $E$ is a $\mathscr{G}_{\delta}$ contained in an $\mathscr{T}_{\sigma}$ of measure 0.

THEOREM 4.3. There exists a continuous subharmonic function $u$ such that $u^{*}$ is defined at each point of $C$ and $E=\left\{\eta \in C: u^{*}(\eta)=-\infty\right\}$, if and only if $E$ is a $\mathscr{G}_{\delta}$ that is not metrically dense in any open arc of $C$.

THEOREM 4.4. Let $\mu$ be a decreasing function on $[0,1)$ such that $\lim _{r \rightarrow 1} \mu(r)=-\infty$. Then there exists a continuous subharmonic function $u$ on $\Delta$ such that

$$
E=\left\{\eta \in C: \limsup _{r \rightarrow 1}[u(r \eta)-\mu(r \eta)]<\infty\right\}
$$

if and only if $E$ is a first-category $\mathscr{T}_{\sigma}$.

We note that since the functions constructed for Theorem B and in $\$ 3$ for Theorem 1.2 are nonvanishing, sufficiency in Theorems 4.1 and 4.4 can be proved using harmonic functions.

5. Concluding remarks. There are a variety of open questions in this subject. There are many important classes of functions for which a radial uniqueness theorem and its converse, or a complete characterization of the boundary zero sets has not been given.

(1) Bounded analytic functions. The classical Riesz radial uniqueness theorem [19] along with the converse of Privalov [18, p. 214] can be stated as follows.

THEOREM E. There exists a nonconstant bounded analytic function $f$ on $\Delta$ such that $f^{*}(\eta)=0$ for each $\eta \in E$ if and only if $E$ has zero measure.

Based on this theorem and elementary topological considerations, it is natural to conjecture that the exact characterization of the boundary zero sets (for nonconstant functions in this class) is the class of $\mathscr{T}_{\sigma \delta}$ sets of measure 0.

A. Samuelsson [21] considered asymptotic radial descent to 0 for bounded analytic functions. He derived a Hausdorff measure condition for sets where this occurs depending on the descent rate to 0 . It is not known if this condition taken together with the topological necessary condition of Theorem 1.2 gives a theorem that is sharp. 
(2) General analytic functions. The Lusin-Privalov radial uniqueness theorem [17, pp. 187-189] and its converse [7] can be given in the following way.

THEOREM F. There exists a nonconstant analytic (or meromorphic) function $f$ on $\Delta$ such that $f^{*}(\eta)=0$ for $\eta \in E$ if and only if for every open arc $A$ of $C$, the set $E$ is not both metrically dense and of second category in $A$.

In this case, the natural conjecture is that the class of boundary zero sets consists of the $\mathscr{T}_{\sigma \delta}$ sets of the type given in the theorem.

(3) Functions having prescribed growth. It is not known whether the converse to the Lusin-Privalov theorem can be proved using functions $f$ such that $|f(z)| \leqslant \nu(|z|)$, where $\nu$ is a prescribed positive continuous function on $[0,1)$ satisfying $\lim _{r \rightarrow 1} \nu(r)$ $=\infty$. Barth and Schneider [3] have shown that there exists a function $f$ with $|f(z)| \leqslant \nu(|z|), z \in \Delta$, such that $f^{*}(\eta)=0$ a.e. in $C$. If Barth and Schneider's result could be modified to insure that for a prescribed first-category set $E$, the function $f$ satisfies $f^{*}(\eta)=0$ for each $\eta \in E$, and $\lim \sup _{r \rightarrow 1}|f(r \eta)|<\infty$ when $\eta \in C \backslash \bar{E}$, then we would have the converse using essentially the same proof as given in [7].

There is a similar question whether the converse in Theorem $\mathrm{C}$ can be proved using functions of prescribed growth.

(4) Univalent functions. Beurling [11] (see also [14, pp. 56-57]) proved that the boundary zero set of a univalent function cannot have positive logarithmic capacity. The converse to this result has not been proved.

(5) Inner functions. Cargo [13] has shown that for a nonconstant inner function $f$, the set $E$ of $\eta \in C$ where $f^{*}(\eta)=1$ is of first category (and measure 0 ). It is not known if there is a converse. If so, there would be different characterizations for the zero sets (see [5]) and the 1-points.

\section{REFERENCES}

1. N. U. Arakeljan, Uniform and tangential approximation with analytic functions, Izv. Akad. Nauk Armjan. SSR Ser. Mat. 3 (1968), no. 45, 273-286. (Russian)

2. M. G. Arsove, The Lusin-Privalov theorem for subharmonic functions, Proc. London Math. Soc. (3) 14 (1964), 260-270.

3. K. F. Barth and W. J. Schneider, On the impossibility of extending the Riesz uniquenss theorem to functions of slow growth, Ann. Acad. Sci. Fenn. AI 432 (1968), 1-9.

4. An asymptotic analog of the F. and M. Riesz radial uniqueness theorem, Proc. Amer. Math. Soc. 22 (1969), 53-54.

5. C. L. Belna, P. Colwell and G. Piranian, Radial limits and distribution by mechanical inheritance, preprint, Univ. of Michigan, Ann Arbor, 1984.

6. R. D. Berman, Elementary proofs of some asymptotic radial uniquenss theorems, Proc. Amer. Math. Soc. 86 (1982), 226-229.

7. A converse to the Lusin-Privalov radial uniqueness theorem, Proc. Amer. Math. Soc. 87 (1983), 103-106.

8. __ The sets of fixed radial limit value for inner functions, Illinois J. Math. 29 (1985), 191-219.

9. Analogues of radial uniqueness theorems for subharmonic functions in the unit disk, J. London Math. Soc. (2) 29 (1984), 103-112.

10. A note on the Lusin-Privalov radial uniqueness theorem and its converse, Proc. Amer. Math. Soc. 92 (1984), 64-66.

11. A. Beurling, Ensembles exceptionnels, Acta Math. 72 (1940), 1-13.

12. R. Cahill, On bounded functions satisfying averaging conditions, Trans. Amer. Math. Soc. 206 (1975), 163-174. 
13. G. T. Cargo, Some topological analogues of the F. and M. Riesz uniqueness theorem, J. London Math. Soc. (2) 12 (1975), 67-74.

14. E. F. Collingwood and A. J. Lohwater, Theory of cluster sets, Cambridge Univ. Press, Cambridge, 1966.

15. J. S. Hwang and P. Lappan, On a problem of Berman concerning radial limits, preprint, Michigan State Univ., 1984.

16. J. E. Littlewood, On functions subharmonic in a circle (II), Proc. London Math. Soc. 28 (1928), $383-394$.

17. N. N. Lusin and I. I. Privalov, Sur l'unicité et la multiplicité des fonctions analytiques, Ann. Sci. École Norm. Sup. 42 (1925), 143-191.

18. I. I. Privalov, Randeigenschaften analytischer Funktionen, VEB Deutscher Verlag der Wissenschaften, Berlin, 1956.

19. F. Riesz and M. Riesz, Über die Randwerte einer analytischen Funktion, Quatrième Congrès des Math. Scand. Stockholm, 1916, pp. 27-44.

20. P. J. Rippon, The boundary cluster sets of subharmonic functions, J. London Math. Soc. (2) 17 (1978), 469-479.

21. A. Samuelsson, On radial zeros of Blaschke products, Ark. Mat. 7 (1968), 477-494.

22. K. F. Tse, An analog of the Lusin-Privalov radial uniqueness theorem, Proc. Amer. Math. Soc. 25 (1970), 310-312.

23. Z. Zahorski, Über die Menge der Punkt in welchen die Ableitung unendlich ist, Tôhoku Math. J. 48 (1941), 321-330.

Department of Mathematics, Wayne State University, Detroit, Michigan 48202 